\title{
METHOD TO INCREASE CLEANING DEGREE OF ELECTROSTATIC AIR CLEANER
}

\author{
Alexander Lysakov, Gennady Nikitenko, Evgeny Konoplev, Vitaly Grinchenko \\ Stavropol State Agrarian University, Russia \\ s_lysakov@mail.ru, nikitenko_gv@mail.ru, konoplev82@mail.ru, grinchen_ko@mail.ru
}

\begin{abstract}
An electrostatic air cleaner is highly efficient for air clearing, which allows blocking dust, tiny particles and bacteria. However, the cost of such devices is quite high; for example, the increase of the degree of cleaning by $6 \%$ raises the relative value almost 3 times. As a result, it restrains their distribution in agriculture. The solution to this problem is possible by upgrading the design of the electrostatic air cleaner, which consists in science-based choosing the area of the collecting electrode. The standard research methods were used for the experiment: the method of multifactorial experiment, statistical analysis, determination of the adequacy of the experimental data. Several collection electrodes have been studied in the form of tubes with different inner surface, so that their area corresponds to the analytical values: smooth surface, corrugated surface, undulated surface, complex surface with interleaving niches and crowns (like cuts in firearms). As a result of the experimental studies the dependence of the degree of cleaning of the electrostatic air cleaner $\eta$ on the speed of the particle movement to the collecting electrode $v$ and supply air $\mathrm{w}$ at a constant square area of the collecting electrode is found. The maximum degree of air cleaning from bacterial pathogens varies from 60 to $99 \%$. The use of air cleaning installations in conjunction with recirculation allows reducing the gas content in the room, where animals are kept, for 6 times; the number of pathogenic bacteria (coliform bacillus, staphylococcus) is reduced 10 times; the amount of the consumed electric power to maintain the microclimate is reduced 2 times; the young stock loss is decreased from $40 \%$ to $5 \%$.
\end{abstract}

Keywords: collecting electrode, electrostatic precipitator, ecology.

\section{Introduction}

Cleaning and disinfection of air are processes, which determine largely the effectiveness of current and future livestock indoor climate systems, both in terms of the creation of the optimal parameters of air environment for animals and birds, as well as to ensure the reduction of energy costs in provision of microclimate $[1 ; 2]$.

Cleaning of livestock indoor air improves greatly the operating conditions of the process equipment and it increases undoubtedly its effectiveness. Thus, in the absence of air supply due to dust deposits, heating efficiency of an air heater is reduced by 40-60\%, and the air supply by fan blowers is reduced by $15-20 \%$. In fact, a year after putting into operation all ventilation equipment should be changed. The air in the agricultural buildings needs to be cleaned and disinfected. It is dictated not only by the requirements of minimum energy consumption and saving of energy resources, but also by the modern requirements of environmental protection against contamination of animal waste [3].

Large livestock farms and poultry farms, where huge population of animals and birds is concentrated on a limited area, are massive sources of environmental pollution of quite large areas (pools). This is due to the fact that intensive methods of animal husbandry and poultry lead to a dramatic increase in accumulation in the indoor air metabolic products (harmful gases, water vapor), as well as to increase of dust and bacterial contamination, which not only adversely affects the productivity of animals and birds, but also pollutes the environment. Air pollutant emissions occupy a significant place among animal waste.

In addition to dust, micro-organisms, harmful gases, spent air of the livestock and poultry houses contains fluff, feather, uneaten food and litter, as well as malodorous substances. Along with wellknown classical gases such as methane, ammonia, hydrogen sulfide, carbon dioxide in the air of the livestock and poultry houses, there are 27 gas compounds contained. Basic smells arise from the presence of hydrogen sulfide, ammonia, indole, skatole and mercaptans.

In pig feeding houses on per 1000 heads basis of animals per day from 2 to $4 \mathrm{~kg}$ of carbon dioxide and ammonia, from 4 to $5 \mathrm{~kg}$ of dust, more than 3-4 billion microbial bodies are removed with the ventilation air. The amount of hydrogen sulfide, which is emitted, sometimes reaches 2-3 $\mathrm{kg}$ per day. In addition, there are significant amounts of methane, amines, mercaptans, skatole, organic acid, amide alcohol and sulfides in the premises. The existing ventilation systems release all of these into the atmosphere and pollute the air basin. 
Despite odor nuisance, these gases have a harmful physical influence not only on animals and poultry, but also on humans. Significantly dusty feed leads to delayed maturation of animals, weight loss, reduced milk production and fertility. Disperse pollution agent, which is passing with air through the airway, causes silicosis, the nitrogen oxides and sulfur cause pulmonary edema. Furthermore, it depends on the kind and amount of pollution agents, which enter the animal with the air, that can be observed as general toxicity, cancers, mutations and other undesirable effects. The small aero suspension and gaseous pollutants are hurtful to the human health $[4 ; 5]$. And also it is more dangerous for a human, when dust is finer, because fine dust penetrates deeply into the respiratory tract. Protective ability of the organism is insufficient in view of ingestion into the lungs of very fine particles (diameter of less than 1 micron), they are deposited primarily in alveolar tissue; up to $70 \%$ of the deposited dust in the lungs of humans and animals, which are affected by silicosis, are composed of such particles [6;7].

The simple means of cleaning are a variety of mechanical dust filters. Dust filter is a special fabric of different fibers that can delay the dust particles from 0,3 microns of size and above. It works quite simple: the fan forces the air through the fabric and thereby frees from the dust particles. The application technology of dust filters in industrial and household air purifiers is widely distributed in Europe and the US and it is called HEPA (High Efficiency Particulate Air). It is a Petryanov's fabric in Russia. The main disadvantage of the dust filter is that it cleans only dust particles of medium dispersion, furthermore, the ultrafine dust concentration in the air due to the forced air movement may even increase [8; 9].

Today there is proposed a large number of combined air cleaning means: photocatalytic filter, carbon filter adsorption, adsorption ozone photo- electrostatic cleaner, but all of them are focused on residential consumers and have insufficient purity for use in livestock enterprises. An electrostatic air cleaner is one of the leaders here, which is capable of trapping particles of a size of 1 micron and above, and to saturate the air with useful negative ions. The wide spread of electrostatic air cleaners is prevented by the applied high voltage (from $10 \mathrm{kV}$ and above) and their high cost, as we can see in Fig. 1, the increase in the cleaning degree is by 6 per cent. It leads to an increase in the relative value nearly 3 times.

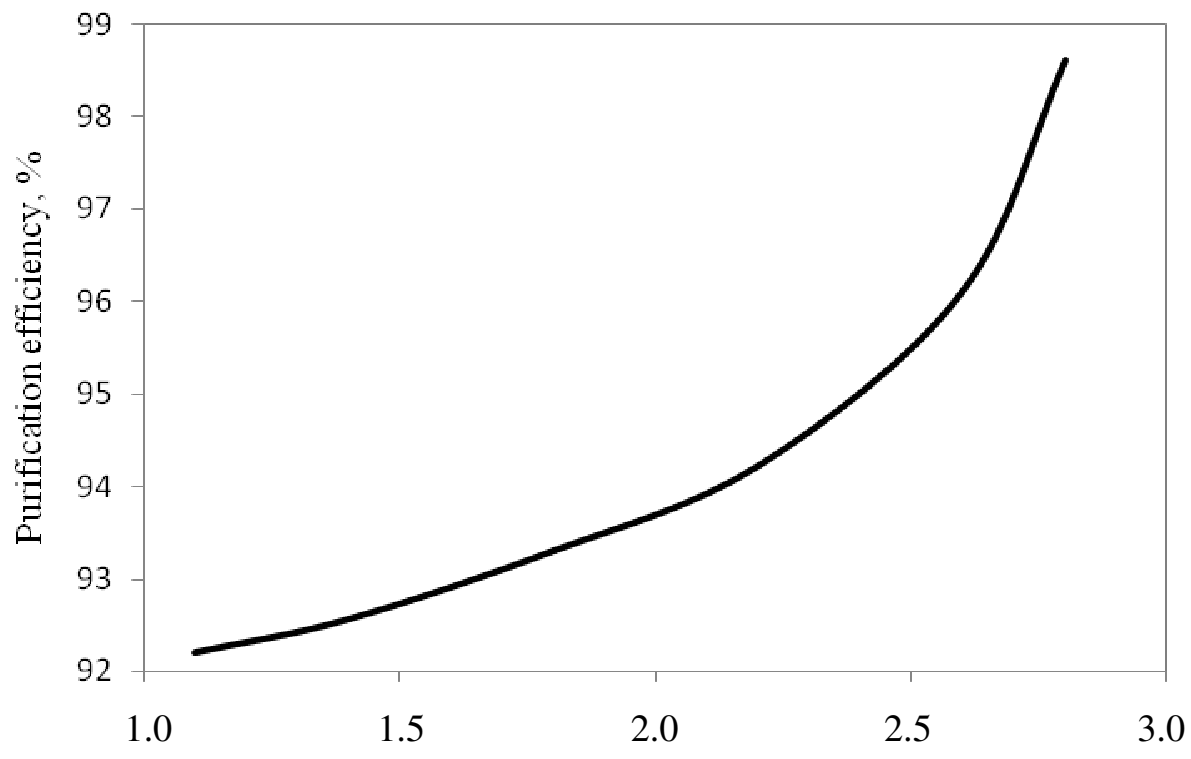

Relative cost of the electrostatic air cleaner

Fig. 1. Dependence of purification efficiency of electrostatic air cleaner on relative cost of electrostatic air cleaner

\section{Materials and methods}

The electrostatic air cleaner structure consists of the corona electrode, to which high voltage is applied, and the collecting electrode, where the particle collection happens. 
There is intense impact ionization gas at the surface of the corona electrode, when a sufficiently large voltage is applied to the interelectrode gap. This corona discharge does not spread on the whole electrode gap and fades with decreasing strength of the electric field in the direction of the collecting electrode. Gas ions of different polarities, which are generated in the crown area under the electric field forces, move toward electrodes of different kind. And thus there is an electric current in the electrode gap, which is called the electric current of corona. The trapped particles due to adsorption of ions on their surface acquire an electric charge in the interelectrode gap, and they move to the electrodes deposited on them under the influence of the electric field strength. The basic amounts of particles are collected on the developed surface of the collecting electrodes, where minority of them falls on the corona electrodes. While collecting on the electrodes, the particles are removed by shaking or by washing the electrodes.

It is known that the cleaning degree of the electrostatic air cleaner depends on a number of factors, which have a significant impact on the quality of the purified air. The most important of these factors are the velocity of the particles to the collecting electrode, the surface area of the collecting electrode and the air supply.

The authors have carried out theoretical studies to determine the nature of the change in the cleaning degree of the electrostatic air cleaner for the following values: velocity to the collecting electrode $v$ equals to $0.5 \mathrm{~m} \cdot \mathrm{s}^{-1} ; 1 \mathrm{~m} \cdot \mathrm{s}^{-1} ; 2 \mathrm{~m} \cdot \mathrm{s}^{-1} ; 3 \mathrm{~m} \cdot \mathrm{s}^{-1} ; 4 \mathrm{~m} \cdot \mathrm{s}^{-1} ; 5 \mathrm{~m} \cdot \mathrm{s}^{-1} ;$ surface area of the collecting electrode $S$ equals to $0.1 \mathrm{~m}^{2} ; 0.5 \mathrm{~m}^{2} ; 5 \mathrm{~m}^{2}$; air supply $\mathrm{w}$ in the electrostatic air cleaner is $0.1 \mathrm{~m}^{3} \cdot \mathrm{s}^{-1}$; $0.5 \mathrm{~m}^{3} \cdot \mathrm{s}^{-1} ; 1 \mathrm{~m}^{3} \cdot \mathrm{s}^{-1} ; 4 \mathrm{~m}^{3} \cdot \mathrm{s}^{-1} ; 8 \mathrm{~m}^{3} \cdot \mathrm{s}^{-1} ; 10 \mathrm{~m}^{3} \cdot \mathrm{s}^{-1}$.

The standard methods of research were used during the experiment such as the multivariate experiment method, statistical analysis and determination of the adequacy of the experimental data. The used measuring equipment was the ammeter, kilovoltmeter and precision balance. All equipment passed certification in the Russian Federation [10; 11].

For experimental practice several collecting electrodes were made in the form of a pipe. They differed by the inner surface, so that their area corresponds to the analytical values such as a smooth surface, corrugated surface, undulated surface, complex surface with alternating recesses and knobs (like cuts in firearms).

In experimental studies the air supply was varied by the fan blower speed, velocity of the particles to the collecting electrode varied by changing the applied voltage to the discharge electrode. In laboratory tests 5 batches of dust, 5 compositions containing carbon of weigh 100 grams were prepared. When the fan blower was switched on at the source of high voltage dust was evenly scattered in the receiver of the electrostatic air cleaner. According to the number of carbon powder, which was on the collecting electrodes and fabric filter as it left air-dust, there was made a comparison with the analytical calculation. Further, a collecting electrode was installed with another surface area, variable voltage and air supply, and then the same experiment was conducted. It was also investigated during the experiments, how the cleaning degree of the electrostatic air cleaner varies in case of emergency change of the supply voltage and at the sudden change in the air flow.

\section{Results and discussion}

The main result of the experimental study was the dependence of the degree of cleaning of the electrostatic air cleaner $\eta$ on the velocity of the particles to the collecting electrode $v$ and air supply w at constant surface area of the collecting electrode, shown in Fig. 2-4. Analysis of the graphs in Fig. 2-4 shows that at constant surface area of the collecting electrode with the increase of the air supply the cleaning degree $\mathrm{w}$ of the electrostatic air cleaner $\eta$ reduced significantly for all variants. The increasing velocity of the particles to the collecting electrode $v$ increases the cleaning degree of the electrostatic air cleaner $\eta$ for all variants. The ratio parameters, for which the degree of cleaning of the electrostatic air cleaner is in the range $\eta=90-100 \%$, considerably increase with the increase of the surface area of the collecting electrode.

For practical purposes of the air cleaning by the electrostatic air cleaners the design factors, at which the degree of cleaning is in the range 90-100, are most interesting. The maximum range of the cleaning degree is observed at a constant surface area of the collecting electrode $S=5 \mathrm{~m}^{2}$. The degree 
of air cleaning is most preferred at varying the air flow $\mathrm{w}$ from $0.1 \mathrm{~m}^{3} \cdot \mathrm{s}^{-1}$ up to $1 \mathrm{~m}^{3} \cdot \mathrm{s}^{-1}$ and the velocity of the particles to the collecting electrode $v$ to $0.5 \mathrm{~m} \cdot \mathrm{s}^{-1}$ from to $5 \mathrm{~m} \cdot \mathrm{s}^{-1}$.

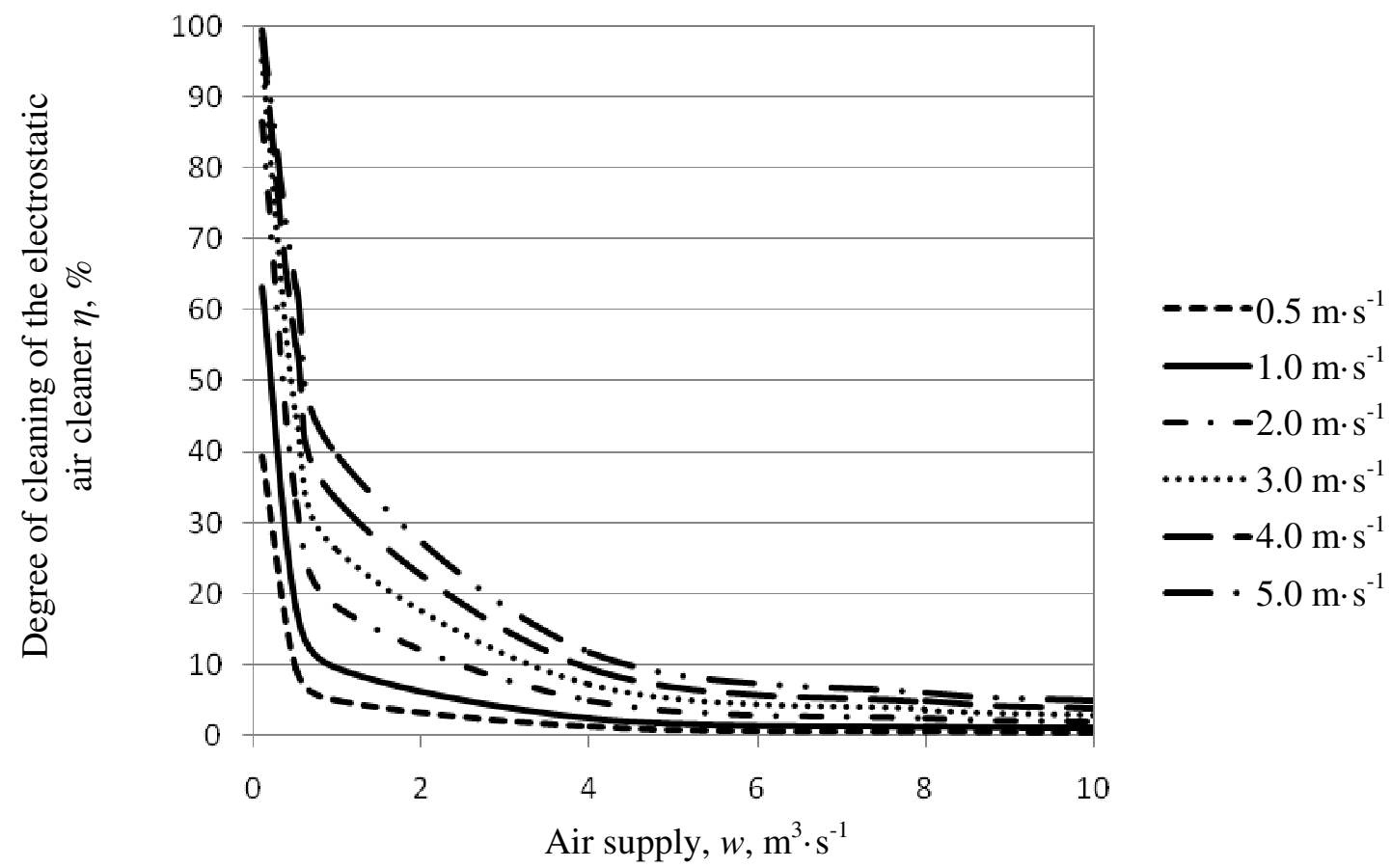

Fig. 2. Dependence of degree of cleaning of electrostatic air cleaner, $\boldsymbol{\eta}$ on velocity of particle to collecting electrode, $v$ (it is shown on the right, $\mathrm{m}^{\cdot-\mathrm{s}^{-1}}$ ) and air supply, $w$ at constant surface area of collecting electrode $S=0.1 \mathrm{~m}^{2}$

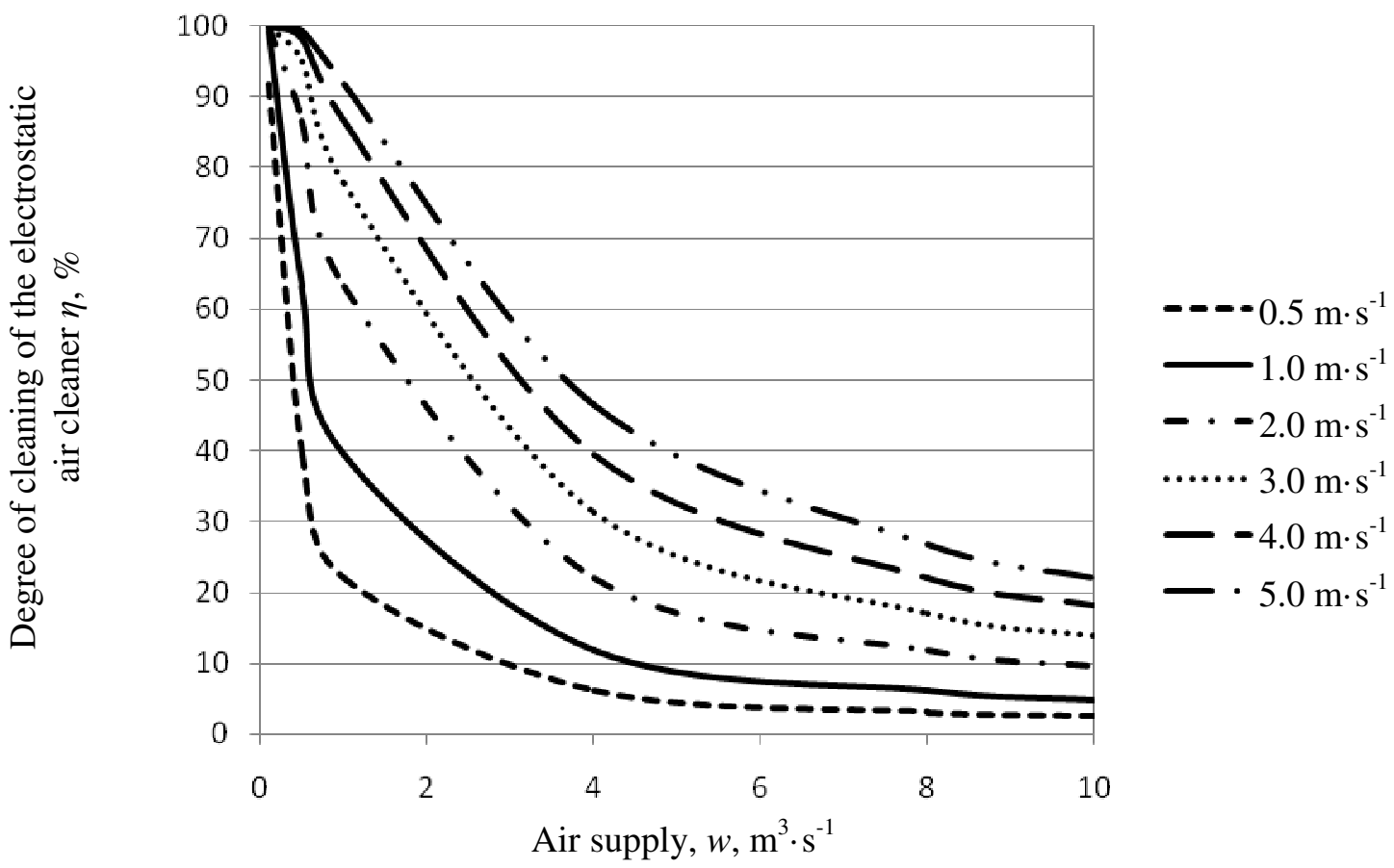

Fig. 3. Dependence of degree of cleaning of electrostatic air cleaner $\boldsymbol{\eta}$ on velocity of particle to collecting electrode $v$ (it is shown on the right, $\mathrm{m} \cdot \mathrm{s}^{-1}$ ) and air supply, $w$ at constant surface area of collecting electrode $S=0.5 \mathrm{~m}^{2}$ 


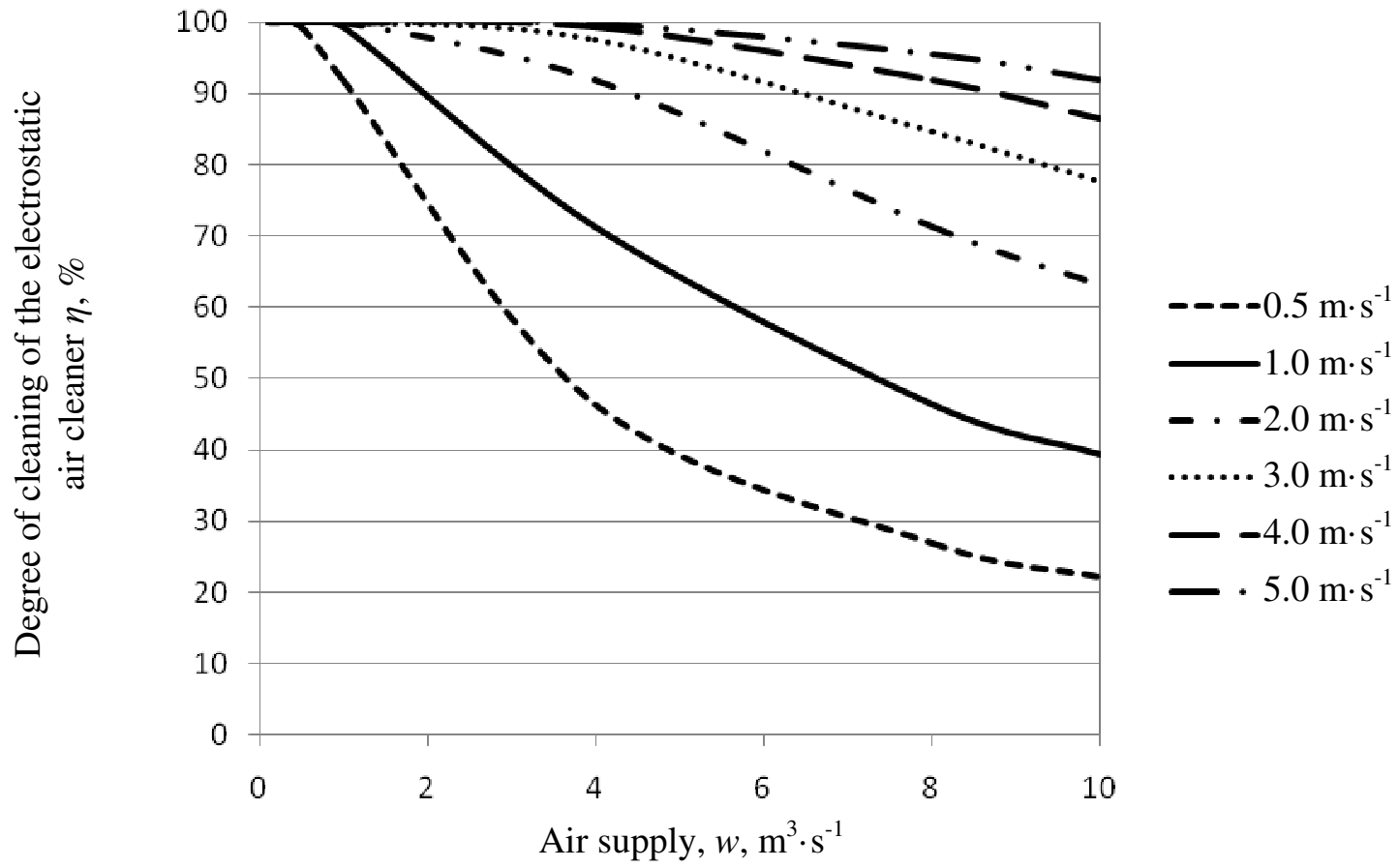

Fig. 4. Dependence of degree of cleaning of electrostatic air cleaner, $\boldsymbol{\eta}$ on velocity of particle to collecting electrode $v$ (it is shown on the right, $\mathrm{m}^{-\mathrm{s}^{-1}}$ ) and air supply $w$ at constant surface area of collecting electrode $S=5 \mathbf{~ m}^{2}$

The experimental studies, which were conducted by the authors together with the Veterinary Service of the farm enterprise "IE Smirnov" in the Kirov Region of the Stavropol Territory, showed that the use of air cleaning installations in conjunction with recirculation allowed to reduce fumes in the room, where animals are kept 6 times; the number of pathogenic bacteria (Escherichia coli, Staphylococcus aureus) is reduced 10 times; the amount of the consumed electric power to maintain the microclimate is reduced 2 times; young loss is decreased from $40 \%$ to $5 \%$.

\section{Conclusions}

1. To increase the cleaning degree of the electrostatic air cleaner it is necessary to increase the velocity of the particles to the collecting electrode.

2. When the air supply is increased, the cleaning degree of the electrostatic air cleaner decreases.

3. The increase of the surface area of the collecting electrode increases the cleaning degree of the electrostatic air cleaner.

4. The experimental studies, which were conducted by the researchers in other countries, showed that the highly cleaning degree of the electrostatic air cleaner (about 90-95\%) allows without significant technical and material costs to improve animal welfare and reduce their morbidity and mortality, which ultimately affects the income of the agricultural enterprise.

\section{Acknowledgements}

The authors express their gratitude to the Head of the farm enterprise "IE Smirnov" Smirnov A.A. for his support in the experimental research.

\section{References}

[1] Wang D., Wang Y., Shi Y., Yu Y. Optimization of casing wall design for electrostatic precipitators. Environmental Engineering and Management Journal, vol. 12(9), 2013, pp. 1879-1883.

[2] Shui T., Shen H., Yang X., Ma H., Nie X., Zhou L., Liu L. PM2.5 filtration performance of electret air filter in office buildings. Chinese Journal of Environmental Engineering, vol. 9(6), 2015, pp. 2933-2940. 
[3] Chang C.-L., Bai H. An experimental study on the performance of a single discharge wire-plate electrostatic precipitator with back corona. Journal of Aerosol Science, vol. 30(3), 1999, pp. 325-340.

[4] Jaworek A., Krupa A., Czech T. Modern electrostatic devices and methods for exhaust gas cleaning: A brief review. Journal of Electrostatics, vol. 65(3), 2007, pp. 133-155.

[5] Katatani A., Hosono H., Murata H., Mizuno A. Collection performance of an electrostatic precipitator using bipolar corona discharges. Proceedings of the 2012 Electrostatic Joint Conference, 2012, Cambridge, ON, Canada, June 12-14, ESA2012 e-proceedings S36, pp. 1-5.

[6] Kim H.-J., Han B., Oda T., Hwang K.-D., Oh W.-S., Yoo S.-Y. Fine particle removal performance of a two-stage wet electrostatic precipitator using a nonmetallic pre-charger. Journal of the Air and Waste Management Association, vol. 61(12), 2011, pp. 1334-1343.

[7] Kuroda Y., Kawada Y., Takahashi T., Ehara Y., Ito T., Zukeran A., Kono Y. Effect of electrode shape on discharge current and performance with barrier discharge type electrostatic precipitator. Journal of Electrostatics, vol. 57 (3-4), 2003, pp. 407-415

[8] Miller J., Hoferer B., Schwab A.J. The impact of corona electrode configuration on electrostatic precipitator performance. Journal of Electrostatics, vol. 44 (1-2), 1998, pp. 67-75.

[9] Mizuno A. Electrostatic precipitation. IEEE Transactions on Dielectrics and Electrical Insulation, vol. 7(5), 2000, pp. 615-624

[10] Grinchenko V., Nikitenko G., Lysakov A., Konoplev E. Milking machine with electropulsator. Proceedings of International conference «Engineering for Rural Development», 2016, Latvia University of Agriculture, pp. 230-235.

[11] Grinchenko V., Nikitenko G., Lysakov A., Konoplev E. Duration of change of pressure in interwall camera of milking cup. Proceedings of International conference «Engineering for Rural Development», 2017, Latvia University of Agriculture, pp. 819-823. 